\title{
Conocimiento sobre tablas estadísticas por estudiantes chilenos de tercer año de Educación Primaria
}

\section{Knowledge of statistical tables by Chilean students third year of primary education}

\author{
Danilo Díaz-Levicoy ${ }^{1}$ \\ Rodolfo Morales ${ }^{2}$ \\ Pedro Arteaga ${ }^{3}$ \\ María del Mar López-Martín ${ }^{4}$
}

\begin{abstract}
Resumen. Esta investigación reporta resultados del conocimiento sobre tablas estadísticas por estudiantes de tercer año de Educación Primaria en Chile. Para la recolección de los datos se diseñó un cuestionario compuesto por tres ítems que abordan actividades relacionadas con las tablas estadísticas incluidas en libros de texto de cursos previos. Este cuestionario se aplicó en tres escuelas municipalizadas de la comuna de San Carlos, región del Bío-Bío en Chile, considerando una muestra intencionada de 79 estudiantes. Los resultados exponen que los estudiantes abordan con mayor éxito las actividades de leer y completar una tabla y, presentan mejores resultados en las actividades donde se exige un nivel de lectura literal que en las centradas en el desarrollo de
\end{abstract}

Fecha de recepción: 16 de diciembre de 2017. Fecha de aceptación: 11 de noviembre de 2018.

1 Departamento Matemática, Física y Estadística, Universidad Católica del Maule, Chile, dddiaz01@ hotmail.com, orcid.org/0000-0001-8371-7899.

2 Sumo Primero en Aula. Comunidad Virtual de Aprendizaje, Pontificia Universidad Católica de Valparaíso, Chile, rodolfo.morales@pucv.cl, orcid.org/0000-0002-1892-1671.

3 Departamento de Didáctica de la Matemática, Universidad de Granada, España, parteaga@ugr.es, orcid. org/0000-0002-8347-7669.

${ }^{4}$ Departamento de Educación, Universidad de Almería, España, mdm.lopez@ual.es, orcid.org/0000-00018677-9606. 
cálculos y comparaciones. Estos resultados evidencian que los estudiantes dominan aspectos básicos del trabajo con tablas estadísticas.

Palabras clave: comprensión, tablas estadísticas, estadística, Educación Primaria.

\begin{abstract}
This paper shows the results of a research on the knowledge of statistical tables by Chilean students of third year of Primary Education in Chile. For data collection, a questionnaire was designed and validated with three items, which address different activities related to statistical tables and which are included in textbooks at previous courses. This questionnaire was applied in three municipal schools in the locality of San Carlos, Bío-Bío region in Chile, considering an intentional sample of 79 students. The results show that students are more successful in reading and completing a table and that they perform better in activities where literal reading is required above activities where the students had to develop calculations and comparisons. These results show that students master basic aspects of working with statistical tables.
\end{abstract}

Keywords: understanding, statistical tables, statistical, Primary Education.

\title{
1. INTRODUCCIÓN
}

En los últimos años, la estadística se ha convertido en un área de gran importancia en diferentes situaciones de nuestra vida cotidiana (científicas, sociales y humanidades). Este hecho viene reflejado por la gran cantidad de información estadística (en forma de tablas, gráficos y resúmenes estadísticos) que se observa en diferentes medios de comunicación (televisión, internet, periódicos, etc.) (Arteaga, Batanero, Cañadas y Contreras, 2011).

En este contexto, las tablas estadísticas son usadas frecuentemente para ordenar información, permitiendo resumir gran cantidad de datos en espacios reducidos (Beltrāo, 2012; Eudave, 2009), razón por la que se consideran como un elemento de la cultura estadística (Cazorla y Utsumi, 2010; Gal, 2002; Watson, 1997). Este término hace referencia al conocimiento que todo ciudadano debe tener para manejar los elementos básicos de la estadística (Batanero, 2004; Wallman, 1993). Además, al igual que los gráficos estadísticos, las tablas son 
consideradas "una de las modalidades específicas de registro y organización de la información útil cognitivamente para una multiplicidad de usos" (Gabucio, Martí, Enfedaque, Gilabert y Konstantinidou, 2010, p. 184).

Desde el punto de vista de las directrices curriculares, diferentes países señalan que, las tablas y los gráficos estadísticos, así como otros temas de estadística y probabilidad, deben ser trabajados desde los primeros cursos de Educación Primaria (e.g., Common Core State Standards Initiative, 2010; Ministerio de Educación, Cultura y Deporte, 2014; National Council of Teachers of Mathematics, 2000). Las bases curriculares para Educación Primaria de Chile (Ministerio de Educación, 2012, 2013a, 2013b) especifican el trabajo con tablas estadísticas desde el primer curso, tal como se observa en la Tabla 1. En el primer curso se deben abordar las tablas de conteo, relacionadas con actividades de lectura y registro de datos e información afín a los estudiantes y su contexto inmediato. Mientras que en el segundo curso las tablas de conteo están asociadas al registro y representación de datos relativos al lanzamiento de dados y monedas. Además, se enfatiza el trabajo con tablas simples para organizar la información de juegos con monedas y dados. El trabajo con estas representaciones se mantiene en los cursos posteriores dentro de la Educación Primaria. Esta investigación se limita a describir los objetivos e indicadores de evaluación relacionados con las tablas estadísticas de los cursos ya realizados por los estudiantes de la muestra.

De acuerdo con estas consideraciones, en esta investigación se plantea el objetivo de analizar el conocimiento que declaran estudiantes de tercer año de Educación Primaria al trabajar actividades sobre tablas estadísticas, que se han seleccionado y adaptado de un estudio previo sobre la presencia de estas representaciones en libros de texto en los dos primeros cursos de Educación Primaria en Chile.

El presente trabajo se ha estructurado en varios apartados: en la sección 2 se exponen los elementos teóricos que han sido empleados, en la sección 3 se recogen algunas de las investigaciones previas desarrolladas en estos niveles educacionales, la metodología del estudio (característica de la muestra y del instrumento de evaluación) se presenta en la sección 4 y en la sección 5 se muestran los principales resultados (a nivel general, según nivel de lectura y por actividad). Para finalizar, la sección 6 presenta las conclusiones más destacadas del estudio. 
Tabla 1. Objetivos e indicadores de evaluación para 1ํy y o de Educación Primaria

\begin{tabular}{|c|c|c|}
\hline Curso & Objetivo & Indicador de evaluación \\
\hline $\begin{array}{l}\text { Primero } \\
\text { (Ministerio de } \\
\text { Educación, 2013a, } \\
\text { p. 105) }\end{array}$ & $\begin{array}{l}\text { Recolectar y registrar datos para } \\
\text { responder preguntas estadísticas } \\
\text { sobre sí mismo y el entorno, usan- } \\
\text { do bloques, tablas de conteo y pic- } \\
\text { togramas. }\end{array}$ & $\begin{array}{l}\text { - Recolectan y organizan datos del en- } \\
\text { torno, usando material concreto y pic- } \\
\text { tórico, registros informales y tablas de } \\
\text { conteo. } \\
\text { - Responden preguntas, utilizando la } \\
\text { información recolectada. }\end{array}$ \\
\hline \multirow[t]{2}{*}{$\begin{array}{l}\text { Segundo } \\
\text { (Ministerio de } \\
\text { Educación, 2013b, } \\
\text { p. 116, 136) }\end{array}$} & $\begin{array}{l}\text { Recolectar y registrar datos para res- } \\
\text { ponder preguntas estadísticas sobre } \\
\text { juegos con monedas y dados, usan- } \\
\text { do bloques, tablas de conteo y picto- } \\
\text { gramas. }\end{array}$ & $\begin{array}{l}\text { - Recolectan datos acerca de lanza- } \\
\text { mientos de dados y monedas. } \\
\text { - Registran datos en una tabla de con- } \\
\text { teo acerca de datos de lanzamientos } \\
\text { de monedas y dados. } \\
\text { - Registran datos acerca de lanzamien- } \\
\text { tos de dados y monedas, usando cu- } \\
\text { bos apilables. } \\
\text { - Responden preguntas en el contexto } \\
\text { de juegos con monedas, usando re- } \\
\text { gistros expresados en cubos apilables. }\end{array}$ \\
\hline & $\begin{array}{l}\text { Registrar en tablas y gráficos de ba- } \\
\text { rra simple, resultados de juegos } \\
\text { aleatorios con dados y monedas. }\end{array}$ & $\begin{array}{l}\text { - Registran resultados de juegos aleato- } \\
\text { rios con dados y monedas en tablas. } \\
\text { - Registran resultados de juegos aleato- } \\
\text { rios con dados y monedas en gráficos } \\
\text { de barra simple. }\end{array}$ \\
\hline
\end{tabular}

\section{MARCO TEÓRICO}

En este apartado exponemos el concepto de tabla estadística y sus elementos, así como los niveles de lectura de Curcio y cols. (Curcio, 1989; Friel, Curcio y Bright, 2001), que permiten describir la actividad cognitiva que se puede realizar con estas representaciones y que es un elemento teórico de nuestro estudio. 


\subsection{TABLAS ESTADÍSTICAS}

Se entienden como un instrumento de transnumeración (Wild y Pfannkuch, 1999), es decir, una forma de obtener una nueva información al cambiar de un sistema de representación a otro. Esta idea se refiere, por ejemplo, del paso de datos (no agrupados) a su recolección en una tabla estadística, permitiendo así observar algunos elementos estadísticos como la moda, el valor máximo, entre otros. Por otro lado, Campbell-Kelly, Croarken, Flood y Robson (2003) y Gabucio et al. (2010) consideran dicho instrumento como un formato de organización gráfica que utiliza un doble eje para cruzar información concerniente a dos conjuntos de categorías o variables, relacionadas y organizadas recíprocamente; y donde cada celda (casilla) representa datos cuantitativos. Posteriormente, EstreIla (2014) define las tablas estadísticas como:

[...] un arreglo rectangular con una estructura que comprende un conjunto de filas y columnas [..., permite presentar los datos correspondientes a una o más variables (características del fenómeno bajo estudio) en forma clasificada y resumida, para permitir la visualización del comportamiento de los datos y facilitar la comprensión de la información que se puede extraer (p. 6).

Estrella (2014) menciona que algunos de los elementos que pueden ser incluidos en las tablas estadísticas son: (a) título: transmite la idea principal sobre la información representada y su contexto; (b) cuerpo de datos: corresponde al bloque rectangular interior compuesto por aquel grupo de celdas formadas por la intersección de filas y columnas; (c) encabezado lateral (primera columna): refleja las categorías de la variable; (d) encabezado superior: presenta el nombre del contenido de las columnas, por ejemplo, frecuencias; (e) totales: relativo a las sumas por fila o columna y/o totales.

\subsection{TIPOS DE TABLAS ESTADÍSTICAS}

Existen diferentes tipos donde, si bien algunos de sus elementos son similares, cada una de ellas tiene características propias. Lahanier-Reuter (2003) describe algunas que son usadas en Educación Primaria, entre las que se destacan: 
Tablas de datos. Son tablas simples, en las que no se trabaja la idea de frecuencia ni de distribución, sino sólo la idea de variable y valor. Un ejemplo es registrar la temperatura durante una semana de una determinada ciudad.

Tablas de frecuencias. Son aquellas en las que se representan las frecuencias (obtenidas mediante agrupación o recuento de datos iguales) asociadas a los valores o categorías de las variables. Ejemplo de esta situación es registrar las edades de los estudiantes de un curso.

Tabla de doble entrada. Tabla en la que se cruzan dos variables, es decir, un valor está relacionado con dos variables a la vez. Situación por la que se consideran como las más complejas. Por ejemplo, representación de las edades de los estudiantes de una clase según su género.

Además de las mencionadas, Mingorance (2014) indica que las tablas de conteo pueden ser trabajadas en el ámbito escolar. Estas tablas son una versión simplificada de las tablas de frecuencia, en las que se realizan recuentos por medio de marcas o símbolos dentro de una misma celda. Un ejemplo es representar con una equis ( $\mathrm{x}$ ) cada vez que un niño menciona sus caricaturas favoritas.

\subsection{NIVELES DE COMPRENSIÓN DE TABLAS ESTADÍSTICAS}

En ocasiones los profesores creen que el trabajo con tablas estadísticas es una actividad fácil para los niños, por lo que no se dedica el tiempo suficiente a su enseñanza y aprendizaje, olvidando que su correcta lectura y construcción moviliza diferentes objetivos matemáticos (Batanero, 2001).

Algunos autores, conscientes de esta complejidad, describen y tratan de caracterizar los diferentes niveles de comprensión para tablas y gráficos estadísticos. Por ejemplo, Curcio (1989) y Friel et al. (2001) proponen diferentes niveles para la lectura de gráficos, que se han adaptado en diferentes investigaciones con tablas estadísticas (e.g., Castellanos, 2013; Díaz-Levicoy, Morales y López-Martín, 2015; Díaz-Levicoy, Sepúlveda, Vásquez y Opazo, 2016; Méndez y Ortiz, 2012; Mingorance, 2014).

A continuación se presentan los cuatro niveles de lectura de gráficos y tablas que van desde el más elemental (lectura literal) al más complejo (análisis críticos de la información) propuestos por Curcio y cols. (Curcio, 1989; Friel et al., 2001), los cuales sirven como marco para dar cuenta del cocimiento, por medio de la comprensión, de estos objetos matemáticos por parte de los estudiantes. 
Nivel 1. Leer los datos. Demanda solo una lectura literal de la información presentada en la tabla, no requiere interpretación. Algunas actividades relacionadas con este nivel son aquellas donde los estudiantes deben leer una frecuencia, una categoría o el título general de la misma.

Nivel 2. Leer dentro de los datos. Pide encontrar un valor por medio de comparaciones u operaciones aritméticas sencillas, además incluye la interpretación de la información contenida en ella. Una actividad relacionada con este nivel consistiría en calcular la cantidad total de estudiantes que participaron en la encuesta, entre otras. Nivel 3. Leer más allá de los datos. Demanda determinar una información ausente en la tabla por medio de predicciones o estimaciones. Ejemplo de una actividad asociada a este nivel sería inferir la temperatura máxima a partir de las temperaturas máximas de una ciudad mostradas en la tabla.

Nivel 4. Leer detrás de los datos. Pide valorar críticamente la manera en que se recogieron los datos, también demanda interpretar la crítica que otras personas hacen del mismo, o bien cuestionar la calidad de los datos. Supone una reflexión del conocimiento matemático y del contexto. Por ejemplo, analizar si la pregunta utilizada para recoger los datos es la apropiada o no.

Por la edad de los estudiantes que son parte de esta investigación y por los resultados de los estudios sobre libros de texto que se describe en el apartado siguiente, solo se utilizarán los dos primeros niveles (leer los datos y leer dentro de los datos).

\section{ANTECEDENTES}

Las investigaciones sobre tablas estadísticas van tomando importancia y asumiendo presencia en la Educación Estadística. Sin embargo, si se remite a trabajos con estudiantes de Educación Primaria se observa que estos son aún escasos. Este hecho puede ser consecuencia, principalmente, del carácter no obligatorio que tenían estos temas en los primeros cursos de Educación Primaria. En lo que sigue, describimos estudios centrados en libros de texto y con estudiantes, ambos en Educación Primaria.

En la línea de los libros de texto, Guimarães, Gitirana, Cavalcanti y Marques (2008) estudian las actividades que usan diferentes representaciones gráficas (gráficos, tablas estadísticas y no estadísticas) de 17 grupos de textos de $1^{\circ}$ a $4^{4}$ 은 de Educación Primaria. Las autoras analizan un total de 2,080 actividades. La 
mayor porción de las actividades alude a las tablas, y de ellas más de la mitad no hacen referencia a la organización de la información. También, observan una distribución no uniforme de las actividades y, además, las que se relacionan con gráficos estadísticos privilegian la interpretación sobre la construcción. En este mismo contexto educativo, Amorim y Silva (2016) analizan cómo se presentan y utilizan las tablas estadísticas en cuatro libros de texto (dos de cuarto y dos de quinto) de Educación Primaria en Brasil. Los resultados muestran que la mayoría de las actividades que hablan de tablas aluden a cuadros y bancos de datos. Se destaca que en tres de los cuatro libros de texto más del $40 \%$ de las actividades se centran en la interpretación de la información mientras que ese porcentaje alcanza niveles muy bajos en relación a las de construcción. Igualmente, se destaca una mayor presencia de datos reales que ficticios, es decir, la mayoría de las tareas están relacionadas con situaciones de la vida cotidiana (por ejemplo, número de habitantes en una ciudad o el sándwich preferido).

En el contexto chileno, Díaz-Levicoy et al. (2015) caracterizan el trabajo con tablas estadísticas propuestas en dos libros de texto de primero y dos de segundo curso de Educación Primaria. Entre los resultados destacan que el tipo de tabla más frecuente son las de conteo, aunque también se observa la presencia de las de frecuencia, datos y doble entrada. Respecto a los niveles de lectura de Curcio y cols. (Curcio, 1989; Friel et al., 2001) solo se encuentran los dos primeros niveles (leer los datos y leer dentro de los datos), con el predominio del nivel 2, asociado a la comparación de información y al desarrollo de cálculos sencillos. También se halla una variedad de actividades, entre ellas: leer (se pide una lectura literal de la tabla); ejemplo (que aclara o define algún concepto o muestra procedimientos); completar (pretende que se finalice la construcción de una tabla); calcular (realizar operaciones o comparaciones con la información de una tabla); traducir (a partir de la tabla construir un gráfico); formular preguntas (indicar la pregunta que permite recoger información de la tabla); explicar (indicar procesos de alguna actividad realizada o justificar la respuesta); recoger datos (aplicar algún instrumento para recoger información). Entre ellas, las más frecuentes son calcular, completar, traducir, ejemplo y leer. Tomando como base estos resultados, se ha diseñado y validado el cuestionario empleado en esta investigación con el fin de estudiar el conocimiento que manifiestan estudiantes de tercer año de Educación Primaria, y cuyo proceso se detalla en la sección de metodología.

Sobre las investigaciones centradas en los niveles de lectura en tablas estadísticas se encuentra la desarrollada por Espinoza (2015) donde se realiza un estudio exploratorio en el contexto peruano. A través de una experiencia de aula, 
la autora analiza cómo un grupo de 15 estudiantes de 1ํ grado de Educación Primaria (6 y 7 años) desarrollan actividades de lectura de información presentada en tablas estadísticas y gráficos de barras. Los resultados muestran que los estudiantes logran leer información directa en ambas representaciones (leer los datos) y responden a preguntas relativas a las cantidades correspondientes de cada una de las categorías de la tabla presentada.

Ochoa (2015), también dentro de un contexto de una experiencia de aula, investiga cómo un grupo de estudiantes peruanos de entre 7 y 8 años de edad desarrollan actividades de completar e interpretar tablas de doble entrada. Los resultados destacan que estos estudiantes son capaces de responder con éxito a preguntas del primer nivel de leer los datos, pero encuentran mayores dificultades en aquellas relacionadas con el nivel leer dentro de los datos. Sin embargo, hay estudios como el de Pereira y Conti (2012) que indican que estudiantes de tercero de Educación Primaria de Brasil responden adecuadamente a preguntas planteadas sobre análisis e interpretación de tablas de doble entrada, específicamente a preguntas donde se realiza una lectura literal y cálculos aritméticos.

Gabucio et al. (2010) aplican un cuestionario para estudiar la comprensión sobre tablas estadísticas en una muestra de 205 estudiantes de $5^{\circ}$ y $6^{\circ}$ de Educación Primaria y de 1ํy yํㅡㄹ de Educación Secundaria de Barcelona (España). Este instrumento considera cuatro aspectos: comprender la estructura tabular, lectura directa de los datos, inferencia de datos e inferencia global. Los resultados muestran que las actividades más sencillas para los estudiantes son las de leer algún dato de la tabla y conocer la estructura de la tabla. Mientras que los de inferencia global son más complejos que los de inferencia a partir de un dato, y estos a su vez más complejos que los de lectura directa. Además, se observa que los ítems más complejos son los más difíciles para los estudiantes de todos los niveles, indiferentes al curso que estos pertenezcan.

Desde otra perspectiva, en el contexto chileno, solo se encuentra el trabajo de Estrella y Olfos (2015), quienes investigan el pensamiento transnumerativo en 80 estudiantes de tercer año de Educación Primaria. Los investigadores indagan sobre las respuestas dadas por los estudiantes a la pregunta “¿De qué manera podemos ordenar y organizar los datos de nuestras colaciones para saber si estamos en riesgo de contraer alguna enfermedad?". Los resultados muestran las técnicas transnumerativas (Chick, 2003) que los estudiantes realizan: ordenamiento, agrupamiento, selección de subconjunto, cambio de tipo de variable, cálculo de frecuencia, graficación y otros cálculos. Solo 5 estudiantes logran construir tablas de frecuencias (de forma básica). 
Con el desarrollo de esta investigación se quiere aportar resultados tanto en el contexto internacional, como en el contexto chileno, dado que son escasos, sobre todo considerando que la inclusión de la estadística en las directrices curriculares de Educación Primaria es relativamente reciente. En concreto, pretendemos ampliar información sobre el conocimiento que presentan los estudiantes al trabajar con estas representaciones.

\section{METODOLOGÍA}

Seguimos una metodología cualitativa (Pérez-Serrano, 1994) y de nivel descriptivo (Hernández, Fernández y Baptista, 2010). El método utilizado para examinar las respuestas entregada por los estudiantes fue el análisis de contenido (LópezNoguero, 2002). Los pasos seguidos en dicho análisis son:

- Elegir el ítem a analizar. Dado que las respuestas de los estudiantes serán transformadas en porciones mínimas de contenido.

- Se establecen, a priori, las categorías que se utilizaran en el análisis de las respuestas de los estudiantes (correctas, parcialmente correctas e incorrectas), las que pueden variar de acuerdo a la codificación de los datos.

- Se codifican las respuestas. Mediante una revisión detallada de las respuestas, estas se relacionan con las categorías definidas a priori, siguiendo un proceso inductivo y cíclico.

- Análisis descriptivo de los datos. Posterior a la codificación y depuraciones de los datos realizamos tablas estadísticas que resumen la información obtenida.

La muestra estuvo compuesta por 79 estudiantes de edades entre 7 y 10 años (Media: 8,25; D. Estándar: 0,65). Estos estudiantes cursaban tercero de Educación Primaria y pertenecían a tres escuelas municipalizadas de la comuna de San Carlos, Provincia de Nuble, en la región del Bío-Bío, distribuida según se muestra en la Tabla 2. La selección de la muestra fue intencional, por la facilidad al acceso a estos centros educativos y la disposición por colaborar con la investigación. A ellos se accedió por medio de la autorización del Alcalde de la llustre Municipalidad de San Carlos, y del Departamento de Administración de Educación Municipal (DAEM). 
Los datos recogidos se han ingresado en una plantilla de Excel, para su posterior análisis.

Tabla 2. Distribución de la muestra de estudiantes

\begin{tabular}{ccc}
\hline Escuelas & Estudiantes & Porcentaje de la muestra \\
\hline 1 & 25 & 31,6 \\
2 & 27 & 34,2 \\
3 & 27 & 34,2 \\
\hline Total & 79 & 100 \\
\hline
\end{tabular}

\subsection{EL INSTRUMENTO DE LEVANTAMIENTO DE DATOS}

La herramienta empleada en la investigación ha sido un cuestionario enfocado en evaluar el conocimiento sobre tablas estadísticas, con actividades extraídas y adaptadas según los resultados de un estudio sobre el tratamiento de este tema, en libros de texto de $1^{\circ}$ y $2^{\circ}$ o de Educación Primaria (Díaz-Levicoy et al., 2015) y validado por juicio de expertos (Díaz-Levicoy, Morales, Cruz y López-Martín, 2016). El cuestionario final quedó conformado por tres ítems, véase Figura 1.

En el primer ítem se presenta una situación, en la que interviene una tabla de conteo, sobre las asignaturas preferidas por estudiantes de un tercer año de Educación Primaria. De acuerdo a la tabla se plantean 3 actividades, donde la pregunta 1 (identificación del título) se corresponde con el nivel 1 de Curcio y cols. (Curcio, 1989; Friel et al., 2001) de leer los datos, mientras que la 2 y 3 exigen un nivel 2 leer entre los datos. Respecto al tipo de actividad, la primera se clasifica como una actividad de leer un dato de la tabla, mientras que la segunda y tercera se clasifican como de calcular (Díaz-Levicoy et al., 2015).

En el segundo ítem se presentan los colores favoritos de un grupo de estudiantes de tercer año de Educación Primaria, en dicho ítem se deben realizar cinco actividades, las dos primeras exigen un nivel 1 de Curcio y cols. (Curcio, 1989; Friel et al., 2001) leer los datos, mientras que las tres siguientes exigen un nivel 2 leer entre los datos. Estas preguntas hacen referencia a actividades como: completar una tabla (actividad 1), leer (actividad 2), calcular (actividad 3, 4 y 5) de acuerdo a como lo describen Díaz-Levicoy et al. (2015). 
Por último, el tercer ítem consta de cuatro actividades: una de completar tabla, dos de leer y una de calcular. Respecto a los niveles de lectura, la actividad 1 implica un nivel de lectura 2 leer dentro de los datos, ya que el estudiante debe contabilizar los iconos asociados a cada fruta y multiplicarlo por 2. Las actividades 2 y 3 piden un nivel 1 de leer los datos, ya que se debe leer una categoría y una frecuencia, respectivamente, con información fácilmente extraíble de la tabla. Del mismo modo la actividad 4 conlleva realizar una suma entre la cantidad de frutillas y naranjas que hay en total, lo que implica realizar una adición con dichos valores.

Si bien estas actividades se han extraído de libros de texto de $1^{\circ}$ y $2^{\circ}$ de Educación Primaria, se ha decidido aplicar a estudiantes de tercer grado, a mediados del primer semestre del curso, para asegurarnos que estos temas hayan sido trabajados en clases, incluso en los casos que los contenidos finales de segundo se hayan trabajo a comienzos de tercero.

De las respuestas entregadas por los estudiantes ( $\mathrm{E} X$, donde $x$ es el número que se asigna al estudiante para la codificación de los datos) realizamos un análisis de contenido, lo que ha permitido clasificarlas de acuerdo a tres grandes grupos de respuestas: correctas (cuando el estudiante hace un uso adecuado de los datos y responde de acuerdo a lo pedido), parcialmente correctas (cuando trabaja correctamente con los datos, pero con pequeños errores al desarrollar algún cálculo o al redactar su respuesta) e incorrectas (cuando la respuesta no está relacionada con lo que se pide y no realiza los cálculos necesarios para llegar a una respuesta correcta o simplemente no responde la actividad). En el apartado de resultados, se detalla cada una de estas categorías, ya que en cada apartado no se puede utilizar el mismo criterio, dado que dependerá del tipo de tarea, su complejidad y las respuestas de los estudiantes. 
ACTIVIDAD 1. Lee la siguiente situación y responde las preguntas.

A un grupo de estudiantes de tercer año básico se les aplicó una encuesta y las respuestas a una de las preguntas se organizaron en la siguiente tabla.

\begin{tabular}{|c|c|c|}
\hline \multicolumn{3}{|c|}{ Asignatura preferida } \\
\hline Asignatura & Conteo & Cantidad de votos \\
\hline Educación Tecnológica & $\| \omega$ & 4 \\
\hline Matemática & 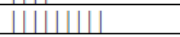 & 9 \\
\hline Educación Física & 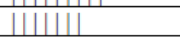 & 7 \\
\hline Ciencias & $\omega \|$ & 6 \\
\hline
\end{tabular}

De acuerdo a la tabla:

1. ¿Cuál es el título de la tabla?

2. ¿Qué asignatura obtuvo la mayor votación?

3. ¿Cuál es la cantidad total de estudiantes que respondieron a la pregunta? Escribe el proceso seguido.

ACTIVIDAD 2. Lee la siguiente situación y desarrolla las actividades.

A cada uno de los estudiantes de tercer año básico se les preguntó por su color favorito. Las respuestas de cada uno de ellos fueron las siguientes:

rojo, azul, verde, rojo, amarillo, rojo, verde, verde, verde, azul, amarillo, rojo, rojo, verde, azul,

azul, azul, verde, rojo, amarillo, azul, azul, amarillo, rojo, rojo

1. Complete la siguiente tabla con la información anterior

\begin{tabular}{|c|c|c|}
\hline \multicolumn{2}{|c|}{ Color preferido } \\
\hline Color & Conteo & Cantidad \\
\hline Rojo & & \\
\hline Azul & & \\
\hline Amarillo & & \\
\hline Verde & & \\
\hline
\end{tabular}

2. ¿Cuántos estudiantes prefieren el color azul?

3. ¿Cuál es el color de mayor preferencia?

4. ¿Cuál es la diferencia entre las cantidades del color de mayor y menor preferencia?

5. ¿Cuántos estudiantes respondieron la pregunta?

ACTIVIDAD 3. Lee la siguiente situación y desarrolla las actividades.

A un grupo de estudiantes de tercer año básico se les realizó una pregunta sobre su fruta preferida cuya información se organizó en el siguiente pictograma. Cada círculo representa el voto de dos estudiantes ( $0: 2$ estudiantes).

1. Complete la tabla

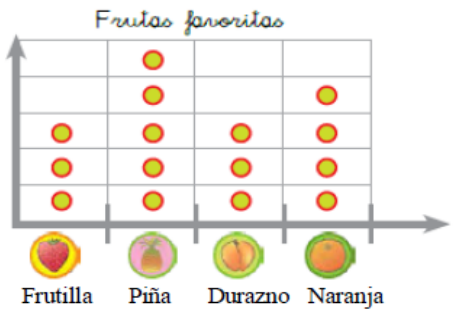

\begin{tabular}{|c|c|}
\hline \multicolumn{2}{|c|}{ Frutas favoritas } \\
\hline Tipo Fruta & Cantidad de votos \\
\hline Frutilla & \\
\hline Piña & \\
\hline Durazno & \\
\hline Naranja & \\
\hline
\end{tabular}

2. Qué fruta fue la favorita solamente por 8 estudiantes?

3. ¿Cuántos estudiantes prefieren la piña?

4. ¿Cuántos estudiantes en total prefieren las frutillas y las naranjas?

Figura 1. Cuestionario de evaluación (Díaz-Levicoy et al., 2015). 


\section{RESULTADOS}

Analizamos la respuesta de los estudiantes a cada una de las actividades de los ítems, clasificándolas como correctas, parcialmente correctas e incorrectas. A continuación mostramos una descripción de cómo consideramos los tres criterios de clasificación y ejemplos representativos en algunos casos. Posteriormente, mostramos un análisis de acuerdo a los niveles de lectura y finalizamos mostrando un resumen del porcentaje de logro por tarea pedida.

\subsection{ANÁLISIS DE LAS RESPUESTAS DEL ÍTEM 1}

Con el fin de analizar las respuestas de cada una de las actividades que constituyen el ítem 1, se han generado las categorías que se presentan en la Tabla 3.

Tabla 3. Codificación de las respuestas a las actividades del ítem 1

\begin{tabular}{|c|c|c|c|}
\hline & Correcta & Parcialmente correcta & Incorrecta \\
\hline Actividad 1.1 & $\begin{array}{l}\text { Escritura del título tal } \\
\text { cual aparece en la tabla }\end{array}$ & & $\begin{array}{l}\text { No se menciona la informa- } \\
\text { ción contenida en el título o } \\
\text { simplemente no responde }\end{array}$ \\
\hline Actividad 1.2 & $\begin{array}{l}\text { Se indica el nombre de la } \\
\text { asignatura que tiene ma- } \\
\text { yor votación }\end{array}$ & $\begin{array}{l}\text { Se mencionan dos cate- } \\
\text { gorías que pueden ser las } \\
\text { de mayor votación y no } \\
\text { discrimina entre ellas }\end{array}$ & No se da respuesta \\
\hline Actividad 1.3 & $\begin{array}{l}\text { Se responde la cantidad } \\
\text { total solicitada }\end{array}$ & $\begin{array}{l}\text { Se mencionan los sujetos } \\
\text { que fueron encuestados, } \\
\text { pero no la cantidad total }\end{array}$ & $\begin{array}{l}\text { No se da respuesta alguna o } \\
\text { se responde con el nombre } \\
\text { de una categoría o la canti- } \\
\text { dad de votos de una de ella }\end{array}$ \\
\hline
\end{tabular}

La Tabla 4 recoge, a nivel general, las respuestas de los estudiantes a este ítem. De la información obtenida se observa que tanto la actividad 1.1 como la actividad 1.2 presentan un índice de logro elevado, 89,9\% y 94,9\% respectivamente. Sin embargo, la actividad 1.3, donde se pide calcular la cantidad total de encuestados de la tabla, ha supuesto mayor dificultad para los estudiantes ya que el $36,7 \%$ de las respuestas no han sido clasificadas como correctas. 
Tabla 4. Distribución de la frecuencia (porcentaje) de los tipos de respuestas en el ítem 1

\begin{tabular}{cccc}
\hline Pregunta del ítem & Correctas & Parcialmente correctas & Incorrectas \\
\hline 1.1 & $71(89,9)$ & $0(0)$ & $8(10,1)$ \\
1.2 & $75(94,9)$ & $1(1,3)$ & $3(3,8)$ \\
1.3 & $50(63,3)$ & $1(1,3)$ & $28(35,4)$ \\
\hline
\end{tabular}

\subsection{ANÁLISIS DE LAS RESPUESTAS DEL ÍTEM 2}

En la primera actividad de este ítem se propone completar una tabla de conteo a partir de un conjunto de datos. En este contexto, las respuestas de los estudiantes permitieron generar las siguientes categorías.

- Correcta. Cuando el estudiante completa las columnas "conteo" y "cantidad" de la tabla con la información otorgada tal como lo hace E23 (Figura 2).

\begin{tabular}{|c|c|c|}
\hline \multicolumn{3}{|c|}{ COLOR PREFERIDO } \\
\hline Color & Conteo & Cantidad \\
\hline Rojo & & 7 \\
\hline Azul & & 7 \\
\hline Amarillo & & 6 \\
\hline Verde & & 6 \\
\hline
\end{tabular}

Figura 2. Respuesta de E23.

- Parcialmente correcta. En esta categoría se han considerado aquellos casos en el estudiante comete algún error al registrar el conteo o la cantidad (recuento). Ejemplo de ello se ve en la Figura 3 donde el estudiante E74 realiza el conteo y recuento de tres de los cuatro colores, faltando solo agregar un verde al que no está marcado en la parte superior de la Figura. 


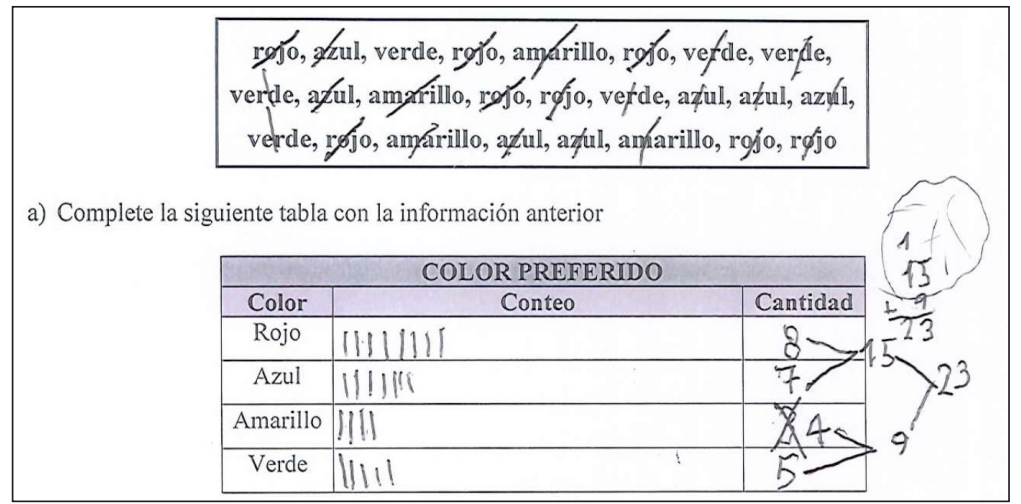

Figura 3. Respuesta de E74.

- Incorrecta. Cuando las columnas conteo y cantidad se completan en su mayoría con cantidades que no corresponden a las solicitadas o, simplemente, no completan la tabla. En la Figura 4 se ve un ejemplo de esta situación, en la que el estudiante E64 realiza un conteo que es insuficiente para determinar las frecuencias correctas.

\begin{tabular}{|c|c|c|}
\hline \multicolumn{3}{|c|}{ COLOR PREFERIDO } \\
\hline Color & Conteo & Cantidad \\
\hline Rojo & 1111 & 4 \\
\hline Azul & 111 & 3 \\
\hline Amarillo & 10 & 1 \\
\hline Verde & 10 & 多 \\
\hline
\end{tabular}

Figura 4. Respuesta de E64.

En la segunda actividad se pide leer la cantidad de preferencias del color azul. Las respuestas de los estudiantes a esta actividad se han categorizado de la siguiente manera:

- Correcta. Cuando el estudiante responde "siete" en símbolo o en palabra, por lo que es capaz de leer la frecuencia asociada a la preferencia del color azul.

- Parcialmente correcta. Cuando el estudiante nombra la cantidad de preferencia del color azul pero añade otra categoría más, tal como lo hace el estudiante E63 (Figura 5), que menciona correctamente la cantidad "siete" y el color correspondiente (azul), pero añade otra categoría (color rojo). 


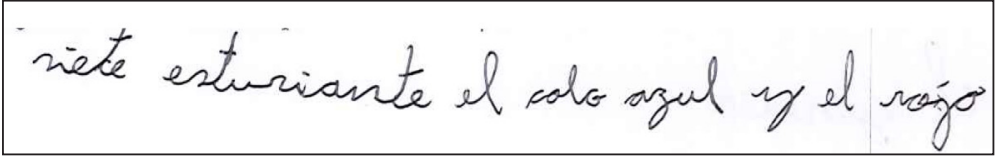

Figura 5. Respuesta del estudiarte E63.

- Incorrecta. Cuando el estudiante responde con una cantidad expresada en símbolo o palabra, o con una de las categorías, diferente a la solicitada, o cuando no responde a la actividad.

En la tercera actividad se propone que los estudiantes identifiquen la categoría que tiene mayor preferencia. Las respuestas de los estudiantes se han categorizado de la siguiente manera:

- Correcta. Cuando el estudiante responde con la categoría "rojo" como color de mayor preferencia, obtenido por medio de la comparación de las frecuencias como lo hace E3.

- Parcialmente correcta. Cuando el estudiante nombra las dos categorías con mayor preferencia o escribe la cantidad asociada a la categoría de la mayor frecuencia. Ejemplo de ello es la respuesta dada por E40 donde indica el valor " 8 " sin asociarlo a su color.

- Incorrecta. Cuando el estudiante nombra una categoría diferente a la de mayor preferencia o cuando el estudiante no responde, tal como lo hace E18 que responde "amarillo".

En la cuarta actividad se propone calcular la diferencia entre las categorías de mayor y menor preferencia. Las respuestas de los estudiantes se han categorizado de la siguiente manera:

- Correcta. Cuando el estudiante calcula la diferencia entre las cantidades de mayor y menor preferencia, y la expresa en palabras o de forma simbólica. En la Figura 6 se ejemplifica la respuesta del estudiante E18 que se ha categorizado como correcta. 


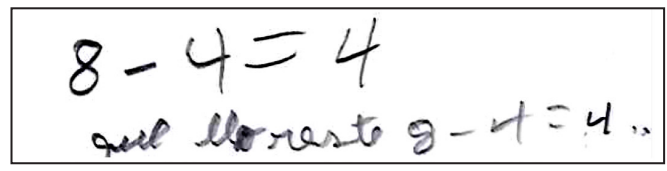

Figura 6. Respuesta del estudiante E18.

- Parcialmente correcta. Cuando el estudiante menciona las categorías de mayor y menor preferencia pero no hace el cálculo correspondiente y no nombra la cantidad de la diferencia. Un ejemplo de ello es la respuesta de E2 que responde "rojo y amarillo", mencionando la categoría de mayor y menor preferencia, pero sin indicar las cantidades que corresponden a cada una de ellas y menos aún su diferencia.

- Incorrecta. Cuando se mencionan las categorías de mayores preferencias y se establece, o no, la diferencia entre las cantidades de ambas; solo menciona la categoría de mayor preferencia; menciona todas las cantidades de todas las categorías; suma todas las cantidades de todas las categorías; menciona las características cualitativas o cuantitativas de las cantidades de mayor preferencia por ejemplo: "uno es alto y el otro es bajo" o "uno es mayor y el otro es menor"; y cuando no responde a la actividad. La Figura 7 muestra una respuesta del estudiante E1, puesto que menciona las categorías con mayores preferencias y sus respectivas cantidades estableciendo la diferencia entre ambas, esta respuesta se ha clasificado como incorrecta.

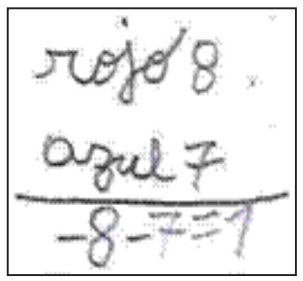

Figura 7. Respuesta del estudiante E1.

En la quinta actividad se pide calcular la cantidad total de estudiantes que respondieron a la pregunta sobre el color preferido. Las respuestas de los estudiantes se han categorizado de la siguiente manera: 
- Respuesta correcta. Cuando el estudiante obtiene 25 como resultado de la suma de los valores de todas las categorías $(8+7+4+6)$, como se observa en la respuesta de E73 (Figura 8).

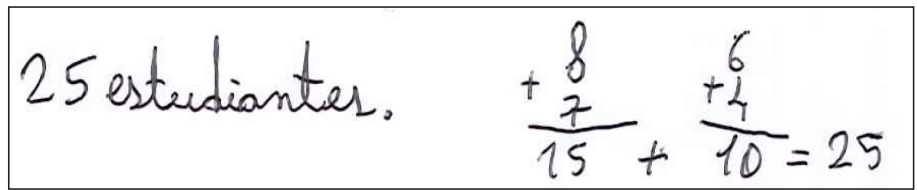

Figura 8. Respuesta del estudiante E73.

- Parcialmente correcta. Cuando el estudiante escribe las cantidades (aunque haya una cantidad diferente a la correcta) de cada una de las categorías en forma de suma, pero no llega a efectuarla o, cuando al efectuar la operación comete algún error de cálculo. Un ejemplo de este tipo de respuesta se muestra en la Figura 9 donde el estudiante E26 escribe las frecuencias en forma de suma, pero no llega a efectuarla, y por tanto no responde a la cantidad total de encuestados.

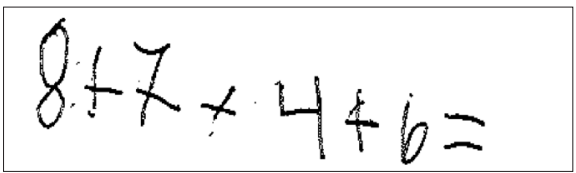

Figura 9. Respuesta del estudiante E26.

- Incorrecta. Cuando el estudiante escribe la suma con los sumandos incorrectos y con un total errado o, cuando entrega un resultado errado sin indicar el procedimiento que ha seguido para llegar a él (Figura 10) o cuando no responde a esta actividad.

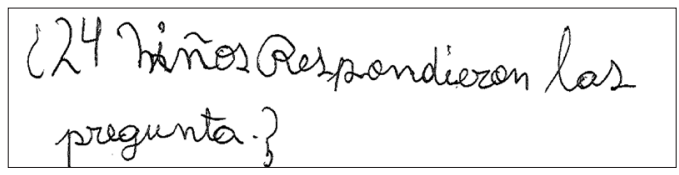

Figura 10. Respuesta del estudiante E30.

A nivel general, como observamos en la Tabla 5, las actividades con mayor índice de logro fueron las actividades 2.2 y 2.3 con porcentajes de 79,7\% y 78,5\% 
respectivamente, no obstante, destacamos la diferencia existente en el porcentaje de respuestas incorrectas entre las actividades 2.2 (19\%) y 2.3 (12,7\%); donde 2.3 tendría mayor logro al considerar las correctas y parcialmente correctas. Finalmente, las actividades 2.4 y 2.5 obtuvieron los menores niveles de logro, asimismo resaltamos el alto porcentaje de preguntas categorizadas como incorrectas (62\% y 45,6\%), considerándose difíciles para los estudiantes dado el nivel de logro que han alcanzado.

Tabla 5. Distribución de la frecuencia (porcentaje) de los tipos de respuestas en el ítem 2

\begin{tabular}{cccc}
\hline Pregunta del item & Correctas & Parcialmente correctas & Incorrectas \\
\hline 2.1 & $54(68,4)$ & $19(24,1)$ & $6(7,6)$ \\
2.2 & $63(79,7)$ & $1(1,3)$ & $15(19)$ \\
2.3 & $62(78,5)$ & $7(8,9)$ & $10(12,7)$ \\
2.4 & $17(21,5)$ & $13(16,5)$ & $49(62)$ \\
2.5 & $34(43)$ & $9(11,4)$ & $43(45,6)$ \\
\hline
\end{tabular}

\subsection{ANÁLISIS DE LAS RESPUESTAS DEL ÍTEM 3}

En la primera actividad se propone a los estudiantes completar una tabla a partir de un pictograma. Las respuestas de los estudiantes se han categorizado de la siguiente manera:

- Correcta. Cuando el estudiante completa la tabla con las cantidades de acuerdo a los convenios de lectura de los pictogramas, es decir, lee la cantidad de iconos y luego multiplica esta cantidad por su valor estadístico (cada icono equivale a 2 estudiantes). La Figura 11 muestra la respuesta correcta del estudiante E19. 


\begin{tabular}{|l|c|}
\hline \multicolumn{2}{|c|}{ Frutas favoritas } \\
\hline Tipo Fruta & Cantidad de votos \\
\hline Frutilla & 6 \\
\hline Piña & -6 \\
\hline Durazno & 6 \\
\hline Naranja & 8 \\
\hline
\end{tabular}

Figura 11. Respuesta del estudiante E19.

- Parcialmente correcta. Cuando el estudiante se equivoca al completar la tabla con alguna de las cantidades. Un ejemplo de esta situación es la respuesta del estudiante E4 (Figura 12) en la que se equivoca con el valor correspondiente a la piña, dado que responde ocho donde su valor correcto es diez.

\begin{tabular}{|l|l|}
\hline \multicolumn{2}{|c|}{ Frutas favoritas } \\
\hline Tipo Fruta & Cantidad de votos \\
\hline Frutilla & 6 \\
\hline Piña & 8 \\
\hline Durazno & 6 \\
\hline Naranja & 8 \\
\hline
\end{tabular}

Figura 12. Respuesta del estudiante E4.

- Incorrecta. Cuando el estudiante completa la tabla con la información literal que aparece en el pictograma. Un ejemplo de respuesta incorrecta es la ofrecida por el estudiante E3 (Figura 13), donde considera que el valor de cada icono es uno, cuando su valor es dos. Dentro de esta categoría se han considerado también aquellas en las que el estudiante responde con cualquier otro número que no se corresponde con el valor de cada icono o simplemente no responde. 


\begin{tabular}{|l|c|}
\hline \multicolumn{2}{|c|}{ Frutas favoritas } \\
\hline Tipo Fruta & Cantidad de votos \\
\hline Frutilla & 3 \\
\hline Piña & 5 \\
\hline Durazno & 3 \\
\hline Naranja & 4 \\
\hline
\end{tabular}

Figura 13. Respuesta de E3.

En la segunda actividad se pide identificar una categoría dada su frecuencia. La categorización realizada ha sido la que se muestra a continuación:

- Correcta. Cuando el estudiante logra leer correctamente la categoría dada su frecuencia. Un ejemplo de respuesta correcta es la del estudiante E78 que explicita "la naranja", para identificar aquella fruta que tiene 8 votos.

- Incorrecta. Cuando el estudiante otorga una categoría diferente a la fruta naranja o cuando no responde a la actividad. Por ejemplo, el estudiante E13 responde "la piña" en lugar de la naranja que tiene ocho preferencias, por tanto, su respuesta se ha categorizado como incorrecta.

En la tercera actividad se solicita leer una frecuencia asociada a una categoría de la tabla. De acuerdo a las respuestas de los estudiantes se definieron las siguientes categorías:

- Correcta. Cuando el estudiante señala la cantidad de votos (diez) correspondiente a la categoría piña.

- Incorrecta. Cuando el estudiante indica cualquier cantidad (simbólicamente o verbalmente) diferente a la correcta. Ejemplo de esta categoría es la respuesta de E58 donde contesta "5 estudiantes". También se han considerado en esta categoría aquellos casos en los que el estudiante no responde a la actividad.

Con respecto a la última actividad, en la que se solicita el cálculo de la cantidad total de votos asignados a dos de las frutas representadas en la tabla (frutillas y naranjas), las categorías definidas han sido: 
- Correcta. Cuando el estudiante señala la cantidad total de votos (14) de ambas categorías. Un ejemplo de ello es la respuesta de E51 (Figura 14), donde además de indicar y sumar las cantidades de las categorías correspondientes a las frutillas y naranjas, señala la cantidad total de preferencias de ambas frutas.

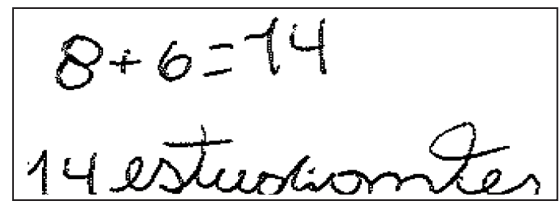

Figura 14. Respuesta del estudiante E51

- Parcialmente correcta. Cuando el estudiante solo menciona las categorías y sus cantidades respectivas, o cuando solo indica las cantidades, sin llegar a calcular el total (Figura 15).

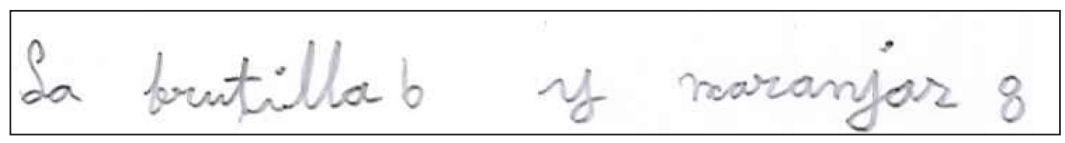

Figura 15. Respuesta del estudiante E22

- Incorrecta. Cuando el estudiante señala las categorías con cantidades diferentes a las correctas; cuando responde con una cantidad diferente a la correcta o cuando no responde a la actividad. Por ejemplo, E6 responde "doce".

En la Tabla 6 mostramos, a nivel general la distribución de las respuestas entregadas por los estudiantes. De la información recogida se observa que, a excepción de la actividad 3.4, los resultados han sido similares; han sido correctamente contestadas aproximadamente por 50\% de estudiantes. Las respuestas incorrectamente alcanzan un porcentaje similar en las cuatro preguntas. 
Tabla 6. Distribución de la frecuencia (porcentaje) de los tipos de respuestas en el ítem 3

\begin{tabular}{cccc}
\hline Pregunta del ítem & Correctas & Parcialmente correctas & Incorrectas \\
\hline 3.1 & $38(48,1)$ & $4(5,1)$ & $37(46,8)$ \\
3.2 & $43(54,4)$ & $0(0,0)$ & $36(45,6)$ \\
3.3 & $41(51,9)$ & $0(0,0)$ & $38(48,1)$ \\
3.4 & $31(39,2)$ & $5(6,3)$ & $43(54,4)$ \\
\hline
\end{tabular}

\subsection{RESUMEN NIVELES DE LECTURA}

En lo que sigue mostramos el estudio de los niveles de lectura alcanzado por los estudiantes en los tres ítems, para ello se ha considerado solo a los estudiantes que responden correctamente a cada pregunta de cada nivel. Las respuestas incorrectas y parcialmente correctas no se han tenido en cuenta dentro de estos niveles, ya que se consideró que lograr algunos de los niveles implica un manejo correcto de los convenidos de lectura y construcción de las tablas, entre ellos, el ser cuidadoso al trabajar con estas representaciones.

Tal y como señalamos en la subsección 2.3 solo se han incluido actividades que demandan los niveles 1 (leer los datos) y 2 (leer dentro de los datos) de Curcio y cols. (Curcio, 1989; Friel et al., 2001), ya que son los únicos niveles que se identificaron en el trabajo de Díaz-Levicoy et al. (2015). Por ejemplo, la respuesta correcta a la primera pregunta del ítem 1 se considera dentro del nivel 1 , ya que el estudiante es capaz de leer correctamente el título de la tabla. En cambio, la respuesta del estudiante E19 (Figura 11) a la primera pregunta del ítem 3 es considerada de nivel 2, porque el estudiante realiza correctamente la lectura la cantidad de iconos y el cálculo de la frecuencia.

La Tabla 7 recoge los porcentajes de logro de cada una de las actividades teniendo en cuenta los dos niveles de lectura. Se observa que las actividades de nivel 1, asociados a la lectura literal de la información de la tabla, es la que los estudiantes responden con mayor facilidad (68,6\%). Sin embargo, con respecto al nivel 2, en el que además de leer alguna información de la tabla se debe realizar algún cálculo o comparación, se obtuvo un menor logro (55,5\%). 
Tabla 7. Distribución del porcentaje de logro según nivel de lectura.

\begin{tabular}{ccc}
\hline Nivel de lectura & Actividad ítem & Porcentaje de logro \\
\hline 1 & 1.1 & 89,9 \\
& 2.1 & 68,4 \\
& 2.2 & 79,7 \\
& 3.2 & 53,2 \\
& 3.3 & 51,9 \\
& Media & 68,6 \\
& 1.2 & 94,9 \\
& 1.3 & 63,3 \\
& 2.3 & 78,5 \\
& 2.4 & 21,5 \\
& 2.5 & 43 \\
& 3.1 & 48,1 \\
& 3.4 & 39,2 \\
& Media & 55,5 \\
\hline
\end{tabular}

\subsection{RESUMEN POR TAREA PEDIDA}

Por último, analizamos los resultados alcanzados por los estudiantes según el tipo de actividad a realizar. Las actividades aquí trabajadas las clasificamos de acuerdo a las establecidas en Díaz-Levicoy et al. (2015). Para esta investigación consideramos las siguientes actividades: leer, calcular, completar y formular preguntas.

De acuerdo a lo mostrado en la Tabla 8, la actividad donde los estudiantes respondieron con mayor facilidad fue leer $(68,7 \%)$, donde se les pidió leer una información puntual de la tabla. En segundo lugar está la actividad de completar (58,3\%), en la que se entregó la estructura de una tabla estadística y el estudiante la debió rellenar mediante la organización de los datos o del cambio de registro (pasar la información de un gráfico a tabla). Le sigue muy de cerca la actividad de calcular (56,7\%), la que conlleva realizar operaciones aritméticas y comparación de información en la tabla. 
Tabla 8. Distribución del tipo de respuesta a tipo de actividad pedida

\begin{tabular}{ccc}
\hline Actividad & Pregunta del ítem & Porcentaje de logro \\
\hline Leer & 1.1 & 89,9 \\
& 2.2 & 79,7 \\
& 3.2 & 53,2 \\
& 3.3 & 51,9 \\
& Media & 68,7 \\
Calcular & 1.2 & 94,9 \\
& 1.3 & 63,3 \\
& 2.3 & 78,5 \\
& 2.4 & 21,5 \\
& 2.5 & 43 \\
& 3.4 & 39,2 \\
& Media & 56,7 \\
& 2.1 & 68,4 \\
& 3.1 & 48,1 \\
& Media & 58,3 \\
\hline
\end{tabular}

\section{DISCUSIÓN Y CONCLUSIÓN}

La reciente inclusión de la estadística y la probabilidad en las directrices curriculares chilenas de Educación Primaria plantea diversos desafíos para el sistema escolar en general. Por un lado, estos contenidos deben ser incluidos en los libros de texto con objeto de que los profesores tengan a su disposición diferentes actividades para desarrollar el proceso de instrucción; por otro, la formación inicial de los profesores de Educación Primaria, debe asegurar que los futuros profesores egresen con las competencias disciplinarias y didácticas para enseñar estos temas; y fomentar la formación continua de los profesores, para completar la formación y entregar nuevas herramientas para utilizar en el aula.

En nuestro caso, nos centramos en las tablas estadísticas, de las cuales ya hemos estudiado su tratamiento en los libros de texto de los dos primeros cursos 
de Educación Primaria (Díaz-Levicoy et al., 2015) y cuyos resultados nos han servido para diseñar y validar un instrumento (Díaz-Levicoy et al., 2016) con el fin de estudiar el conocimiento que tienen los escolares chilenos de tercer año sobre estas representaciones.

Los resultados muestran que en el caso del ítem 1 los estudiantes abordan con mayor éxito la lectura del título de la tabla y el cálculo de la moda. Al considerar las respuestas según el nivel de lectura de Curcio y cols. (Curcio, 1989; Friel et al., 2001), vemos que, en promedio, los mejores resultados son obtenidos en el nivel más elemental leer los datos, situación similar a los resultados de Espinoza (2015) y Ochoa (2015) donde este nivel es alcanzado con facilidad por estudiantes de primeros cursos de Educación Primaria.

En el segundo ítem, formado por cinco actividades, la muestra de estudiantes aborda con mayor facilidad leer información de la tabla y calcular (la moda), mientras que, en otras actividades de calcular, en las que se debe determinar la suma o la diferencia entre frecuencias, conllevan un mayor porcentaje de fracaso, en especial en esta última en que $62 \%$ de los estudiantes no responden la actividad o lo hacen mal. Respecto a los niveles de lectura, los resultados coinciden con los de Gabucio et al. (2010) y Pereira y Conti (2012), donde los estudiantes responden a actividades relacionadas con los niveles 1 y 2 de Curcio, aunque con más dificultades en este último.

Finalmente, en el tercer ítem es donde observamos mayores dificultades para los estudiantes en sus cuatro actividades planteadas. Uno de los principales errores, además de los cálculos, es cuando los estudiantes traducen la información de un pictograma a una tabla de frecuencias, donde el icono usado en el pictograma es diferente de la unidad (vale dos en este caso) y los estudiantes asumen que cada icono es igual a la unidad. Dificultad que Díaz-Levicoy, Arteaga y Batanero (2017) observan en estudiantes de 6으 y 7으 de Educación Primaria, por lo que existe el temor de que estas dificultades se mantengan con el paso de los años.

A nivel general, este trabajo muestra que los estudiantes chilenos de $3^{\circ}$ de Educación Primaria, que fueron objeto de investigación, dominaron aspectos básicos de la lectura e interpretación de las tablas estadísticas, como la lectura literal de información y el desarrollo de cálculos sencillos, coincidiendo con estudios previos en otros contextos. Al considerar las respuestas según el tipo de actividad, vemos que estos tienen mejores resultados en leer y completar tablas que, en calcular lo que se puede justificar por los objetos matemáticos que intervienen en el desarrollo. 
Respecto a los niveles de lectura, vemos la tendencia de que las actividades con mayor éxito son las del nivel 1 (leer los datos), pero con evidencia de que igual las actividades de nivel 2 (leer dentro de los datos) pueden ser abordadas con éxito por los estudiantes.

Por lo anterior, se puede confirmar que las tablas estadísticas se están trabajando en clase y que los estudiantes las comprenden. Sin embargo, creemos que los resultados pueden mejorar mediante metodologías que permitan trabajar con datos que surjan de los intereses de los propios estudiantes y que les resulten motivadores, ya que si bien las actividades de libros de texto chilenos se presentan en contextos próximos a los estudiantes, son escasas las oportunidades donde ellos recojan sus propios datos. Una metodología recomendable en el ámbito de la estadística, y que permite trabajar diversos temas del área y con diferente profundidad, es el trabajo con proyectos (Batanero y Díaz, 2011).

Una instrucción adecuada puede evitar que errores detectados en estos cursos se mantenga en el tiempo, situación preocupante ya que incluso profesores en formación de Educación de Infantil y Primaria presentan problemas al trabajar con estas representaciones en el contexto chileno (e.g., Rodríguez-Alveal y Sandoval, 2012; Díaz-Levicoy et al., 2016).

\section{AGRADECIMIENTOS}

PID2019-105601GB-I00 (MICIN), FCT-16-10974 y Grupo FQM126 (Junta de Andalucía).

\section{REFERENCIAS}

Amorim, N. D. y Silva, R. L. (2016). Apresentaçāo e utilizaçāo de tabelas em livros didáticos de matemática do $4^{\circ}$ e $5^{\circ}$ anos do ensino fundamental. Revista de Educação Matemática e Tecnológica Iberoamericana, 7(1), 1-21.

Arteaga, P., Batanero, C., Cañadas, G. y Contreras, J. M. (2011). Las tablas y gráficos estadísticos como objetos culturales. Números. Revista de Didáctica de las Matemáticas, 76, 55-67.

Batanero, C. (2001). Didáctica de la estadística. Universidad de Granada.

Batanero, C. (2004). Los retos de la cultura estadística. Yupana, 1(1), 27-37

Batanero, C. y Díaz, C. (Eds.) (2011). Estadística con proyectos. Universidad de Granada. 
Beltrāo, T. M. S. (2012). Uma análise da transposição didática externa com base no que propōem documentos oficiais para o ensino de gráficos estatísticos. Revista Paranaense de Educaçāo Matemática, 1(1), 131-152.

Campbell-Kelly, M., Croarken, M., Flood, R. y Robson, E. (Eds.) (2003). The history of mathematical tables. From sumer to spreadsheets. Oxford University Press.

Castellanos, M. (2013). Tablas y gráficos estadísticos en pruebas SABER-Colombia (Trabajo de fin de máster). Universidad de Granada.

Cazorla, I. y Utsumi, M. C. (2010). Reflexōes sobre o ensino de estatística na educaçāo básica. En I. Cazorla y E. Santana (Eds.), Do tratamento da informação ao letramento estatístico (pp. 9-18). Via Litterarum.

Chick, H. (2003). Tools for transnumeration: Early stages in the art of data representation. En R. Bragg, C. Campbell, G. Herbert y J. Mousley (Eds.), Mathematics Education Research: Innovation, Networking, Opportunity. Proceedings of the 23rd annual conference of the Mathematics Education Research Group of Australasia (pp. 167-174). MERGA.

Common Core State Standards Initiative (2010). Common Core State Standards for Mathematics. National Governors Association for Best Practices and the Council of Chief State School Officers.

Curcio, F. (1989). Developing graph comprehension. National Council of Teachers of Mathematics.

Díaz-Levicoy, D., Arteaga, P. y Batanero, C. (2017). Chilean primary school student's levels in reading pictograms. En T. Dooley y G. Gueudet (Eds.), Proceedings of the Tenth Congress of the European Society for Research in Mathematics Education (pp. 740 747). DCU Institute of Education and ERME.

Díaz-Levicoy, D., Morales, R., Cruz, A. y López-Martín, M. M. (2016). Validación de un cuestionario para evaluar la comprensión sobre tablas estadísticas en Educación Primaria. En Escarbajal, A. (Ed.), Libro de actas del IV Congreso Internacional de Investigación e Innovación en Educación Infantil y Educación Primaria (pp. 518-524). Universidad de Murcia.

Díaz-Levicoy, D., Morales, R. y López-Martín, M. M. (2015). Tablas estadísticas en libros de texto chilenos de $1^{\circ}$ y $2^{\circ}$ año de Educación Primaria. Revista Paranaense de Educaçāo Matemática, 4(7), 10-39.

Díaz-Levicoy, D., Sepúlveda, A., Vásquez, C. y Opazo, M. (2016). Lectura de tablas estadísticas por futuras maestras de Educación Infantil. Educaçāo Matemática Pesquisa, 18(3), 1099-1115.

Espinoza, N. (2015). Tablas y gráficos de barras a través del ciclo del pensamiento estadístico. Un estudio con alumnos de primer grado de Educación Primaria (Tesis de Magíster). Pontificia Universidad Católica del Perú. 
Estrella, S. (2014). El formato tabular: una revisión de literatura. Revista Actualidades Investigativas en Educación, 14(2), 1-23.

Estrella, S. y Olfos, R. (2015). Transnumeración de los datos: el caso de las tablas de frecuencia. En P. Scott y Á. Ruíz (Eds.), Educación Matemática en las Américas: 2015. Estadística y Probabilidad (pp. 220-225). Comité Interamericano de Educación Matemática.

Eudave, D. (2009). Niveles de comprensión de información y gráficas estadísticas en estudiantes de centros de educación básica para jóvenes y adultos de México. Educación Matemática, 21(2), 5-37.

Friel, S., Curcio, F. y Bright, G. (2001). Making sense of graphs: critical factors influencing comprehension and instructional implications. Journal for Research in Mathematics Education, 32(2), 124-158.

Gabucio, F., Martí, E., Enfedaque, J., Gilabert, S. y Konstantinidou, A. (2010). Niveles de comprensión de las tablas en alumnos de primaria y secundaria. Cultura y Educación, 22(2), 183-197.

Gal, I. (2002). Adult's statistical literacy: Meaning, components, responsibilities. International Statistical Review, 70(1), 1-25.

Guimarāes, G., Gitirana, V., Cavalcanti, M. y Marques, M. C. M. (2008). Análise das atividades sobre representaçōes gráficas nos livros didáticos de matemática. En V. Gitirana, F. Bellemain y V. Andrade (Eds.), Anais do 2o Simpósio Internacional de Pesquisa em Educaçāo Matemática (pp. 1-12). Universidad Federal de Pernambuco.

Hernández, R., Fernández, C. y Baptista, P. (2010). Metodología de la investigación. McGraw Hill.

Lahanier-Reuter, D. (2003). Différents types de tableaux dans l'enseignement des statistiques. SPIRALE. Revue de Recherches en Éducation, 32, 143-154.

López- Noguero, F. (2002). El análisis de contenido como método de investigación. XXI. Revista de Educación, 4, 167-180.

Méndez, M. y Ortiz, M. (2012). Construcción y lectura de gráficos y tablas estadísticas en tesis de la licenciatura en Psicología Educativa de la Universidad Pedagógica Nacional (Tesis de licenciatura). Universidad Pedagógica Nacional Ajusco.

Mingorance, C. (2014). La estadística en las pruebas de diagnostico andaluzas (Trabajo fin de grado). Universidad de Granada.

Ministerio de Educación (2012). Matemática educación básica. Bases curriculares. Unidad de Currículum y Evaluación.

Ministerio de Educación (2013a). Matemática. Programa de estudio primer año básico. Unidad de Currículum y Evaluación.

Ministerio de Educación (2013b). Matemática. Programa de estudio segundo año básico. Unidad de Currículum y Evaluación. 
Ministerio de Educación, Cultura y Deporte (2014). Real Decreto 126/2014, de 28 de febrero, por el que se establece el currículo básico de la Educación Primaria. MECD.

National Council of Teachers of Mathematics (2000). Principles and standards for school mathematics. NCTM.

Ochoa, E. (2015). El ciclo de investigación del pensamiento estadístico relacionado con tablas de doble entrada. Un estudio con alumnos del segundo grado de Educación Primaria (Tesis de Magíster no publicada). Pontificia Universidad Católica del Perú.

Pereira, E. L. y Conti, K. C. (2012). Interpretando tabelas e construindo gráficos com alunos do $3^{\circ}$ ano do Ensino Fundamental. En M. Tommasiello, A. Marin, S. Pimenta, L. Carvalho y J. Fusari (Eds.), Didática e práticas de ensino na realidade escolar contemporânea: constataçōes, análises e proposiçōes (pp. 5294-5302). Junqueira \& Marin.

Pérez-Serrano, G. (1994). Investigación cualitativa. Retos e interrogantes. I. Métodos. La Muralla.

Rodríguez-Alveal, F. y Sandoval, P. (2012). Habilidades de codificación y descodificación de tablas y gráficos estadísticos: un estudio comparativo en profesores y alumnos de pedagogía en Enseñanza Básica. Avaliaçāo. Revista da Avaliaçāo da Educaçāo Superior, 17(1), 207-235.

Watson, J. (1997). Assessing statistical literacy through the use of media surveys. En I. Gal y J. B. Garfield (Eds.), The assessment challenge in statistics education (pp. 107121). IOS Press.

Wild, C. y Pfannkuch, M. (1999). Statistical thinking in empirical enquiry. International Statistical Review, 67(3), 223-265.

Wallman, K. K. (1993). Enhancing statistical literacy: Enriching our society. Journal of the American Statistical Association, 88(421), 1-8.

\section{DANILO DÍAZ-LEVICOY}

Dirección: Departamento Matemática, Física y Estadística, Facultad de Ciencias Básicas Centro de Investigación en Educación Matemática y Estadística (CIEMAE) Universidad Católica del Maule, Avda. San Miguel 3605, Talca, Chile

Teléfono: (+56) 978065371 